\title{
POLYNOMIAL APPROXIMATIONS OF EXPONENTIAL INTEGRALS
}

\author{
S. R. DRAYSON \\ High Altitude Engineering Laboratory, Department of Aerospace Engineering, \\ The University of Michigan. Ann Arbor, Michigan 48105
}

(Received 23 Mav 1968)

\begin{abstract}
A method is described to calculate polynomial approximations for exponential integrals. Tables of coefficients are presented for exponential integrals of the second, third and fourth order, which allow their rapid evaluation to an absolute accuracy of about $1 \times 10^{-7}$.
\end{abstract}

IN RADIATIVE transfer problems angular integration of the transfer equations frequently produces terms involving exponential integrals. The $n$-th order exponential integral $E_{n}(x)$ is defined by the relation

$$
E_{n}(x)=\int_{1}^{\infty} \mathrm{e}^{-x t} / t^{n} \mathrm{~d} t .
$$

Here $E_{1}(x)$ may be evaluated in a number of ways, including series and asymptotic expansions, or less precisely using polynomial or rational approximations. ${ }^{(1)} \mathrm{A}$ recurrence relation may be used to obtain higher order integrals from $E_{1}(x)$.

The rational approximations are suitable for use in a computer subroutine, but they involve the evaluation of either the exponential function or natural logarithm, or both (depending on the value of the argument $x$ ), a factor which considerably increases computing time. Moreover, values obtained by the recurrence relation for $x>5$ are subject to considerable error. For some applications it is important to have an accurate fast method of evaluating the integrals, and for this reason a series of polynomial approximations has been devised.

Suppose a polynomial of degree $m$

$$
p_{m}(x)=\sum_{i=0}^{m} A_{i} x^{i}
$$

is used to approximate $E_{n}(x)$ on a subinterval $\left(x_{1}, x_{2}\right)$ of the positive $x$-axis, using a least squares technique to determine the coefficients. The condition to be satisfied is that

$$
\int_{x_{1}}^{x_{2}}\left[p_{m}(x)-E_{n}(x)\right]^{2} \mathrm{~d} x
$$


is a minimum. In practice $p_{m}(x)$ is expressed in terms of Legendre polynomials $P_{i}(x)$, orthogonal on $\left(x_{1}, x_{2}\right)$, viz.

$$
p_{m}(x)=\sum_{i=0}^{m} B_{i} P_{i}(x)
$$

The least squares condition gives

$$
B_{i}=\frac{\int_{x_{1}}^{x_{2}} P_{i}(x) E_{n}(x) \mathrm{d} x}{\int_{x_{1}}^{x_{2}}\left(P_{i}(x)\right)^{2} \mathrm{~d} x} \quad i=0,1, \ldots, m .
$$

The integral in the numerator may be expressed as a linear sum of integrals of the form

$$
d_{j}=\int_{x_{1}}^{x_{2}} x^{j} E_{n}(x) \mathrm{d} x \quad j=0,1, \ldots i .
$$

By successive integrations by parts, or by induction on $j$, it can be shown that

$$
d_{j}=\sum_{k=0}^{j} \frac{(j) !}{(j-k) !}\left[x_{1}^{j-k} E_{n+1+k}\left(x_{1}\right)-x_{2}^{j-k} E_{n+1+k}\left(x_{2}\right)\right] .
$$

This expression was used for values of $x_{1}$ greater than about 10. Values of $E_{1}(x)$ were obtained from highly accurate tables, ${ }^{(2,3)}$ and the recurrence relation employed for higher order integrals, using double precision arithmetic.

For small values of $x_{1}$ and $x_{2}$ the numerical values of the terms in the sum are many orders of magnitude greater than $d_{j}$, resulting in severe loss of accuracy. For $x_{1}$ and $x_{2}$ between 0.0 and 1.0 , the values of $d_{j}$ were obtained by Gaussian quadurate of the integral. The exponential integrals were accurately obtained from the series expansion ${ }^{(1)}$ of $E_{1}(x)$. which converges rapidly on this interval, followed by the recurrence relation.

Radiative transfer applications generally require a high absolute accuracy for the exponential integral. Approximations were obtained for $E_{4}, E_{3}$ and $E_{2}$ giving an absolute accuracy of about $1 \times 10^{-7}$. The intervals and polynomial coefficients are given in the accompanying tables. For $x>14.0$ the value of the exponential integrals is taken as zero. A computer capable of representing about eight significant decimal digits is required to maintain full accuracy.

Near $x=0 \cdot 0$, the slope of $E_{2}(x)$ becomes very large, making polynomial approximations less accurate in this region. In the interval $(0.0,0.005)$ the function was evaluated by the expression

$$
E_{2}(x) \sim 1+(y-1+\ln x) x-\frac{1}{2} x^{2}+\frac{1}{12} x^{3}
$$

where $;-0.577215665$ is Euler's constant. 
Polynomial approximations of exponential integrals

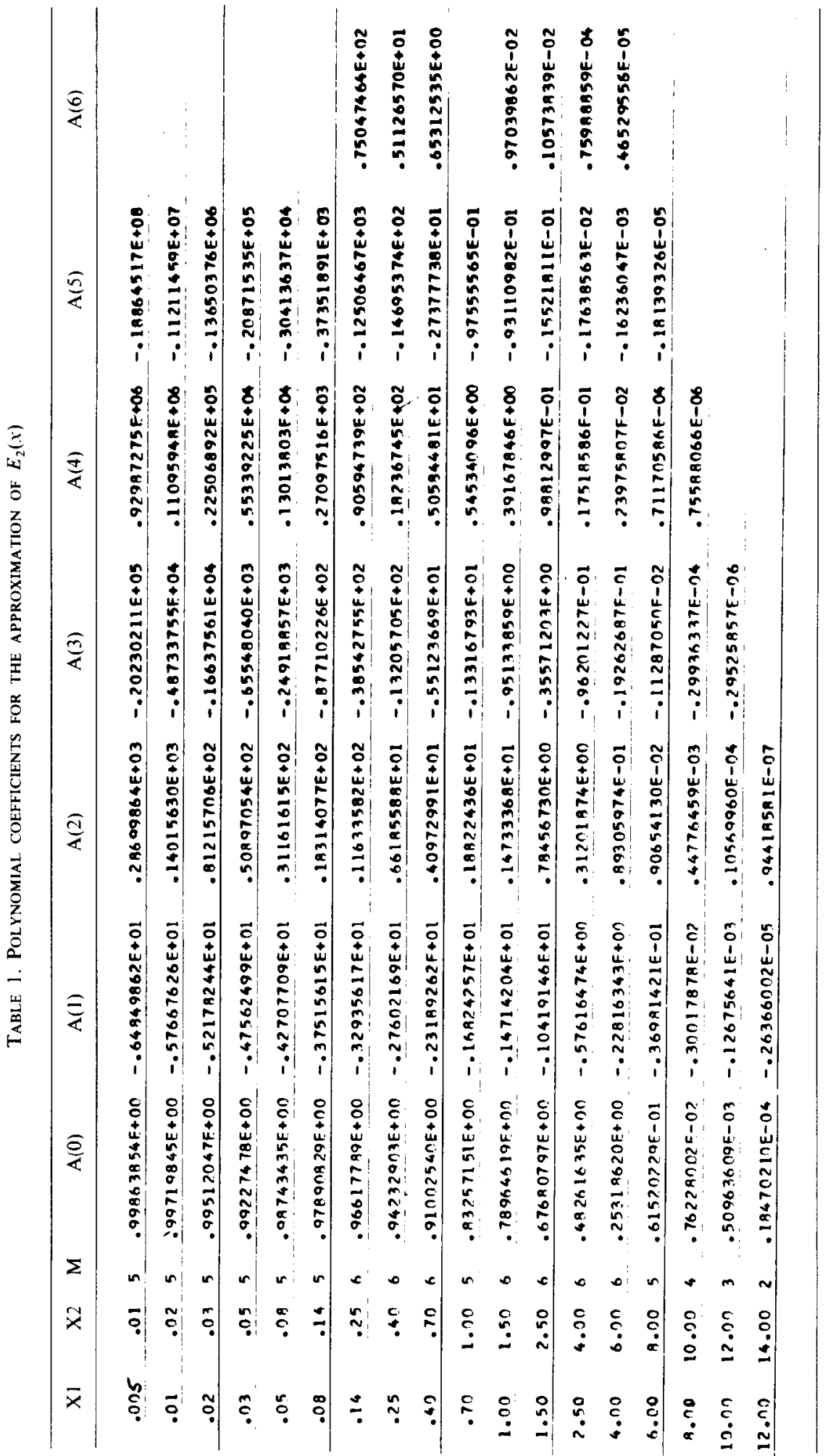




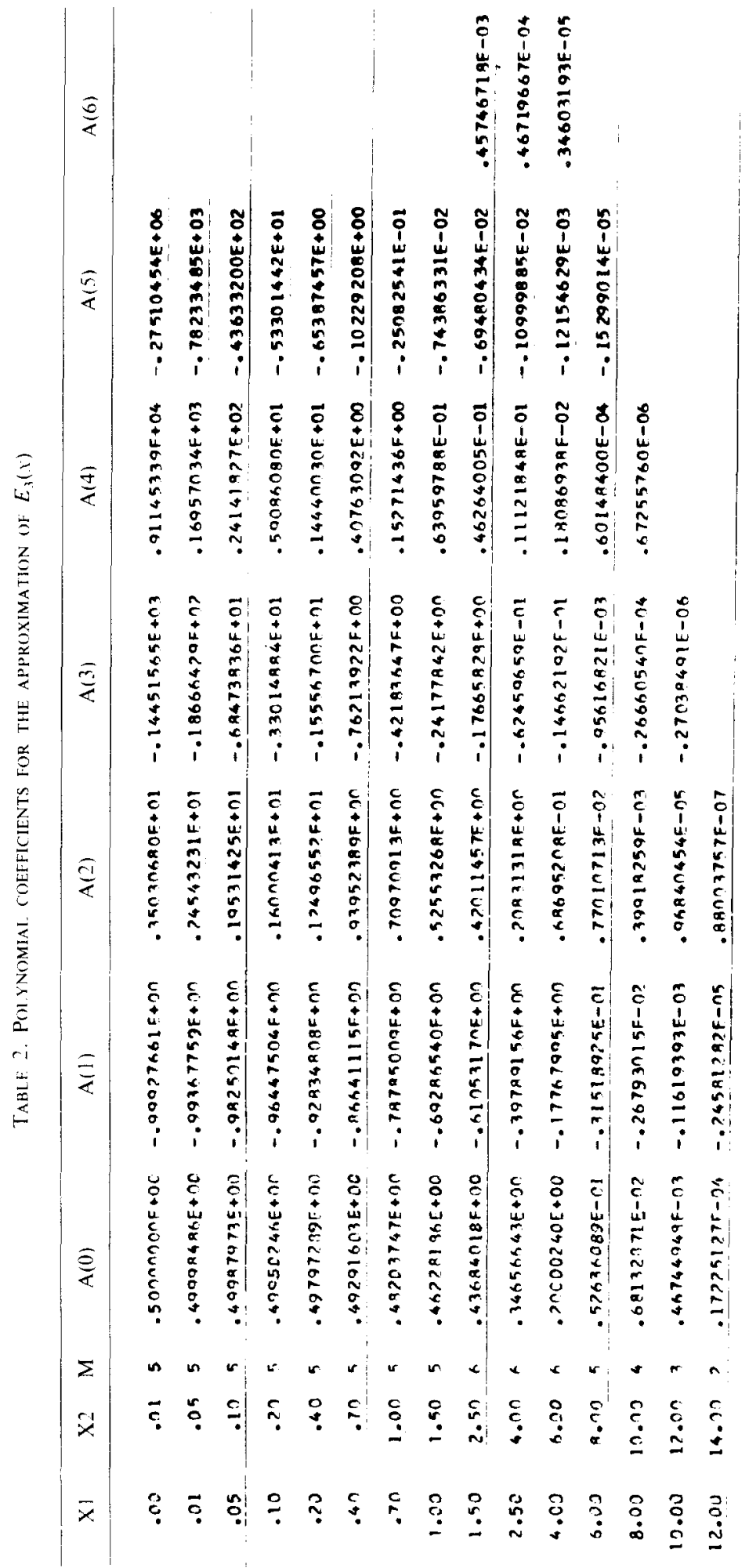


Polynomial approximations of exponential integrals

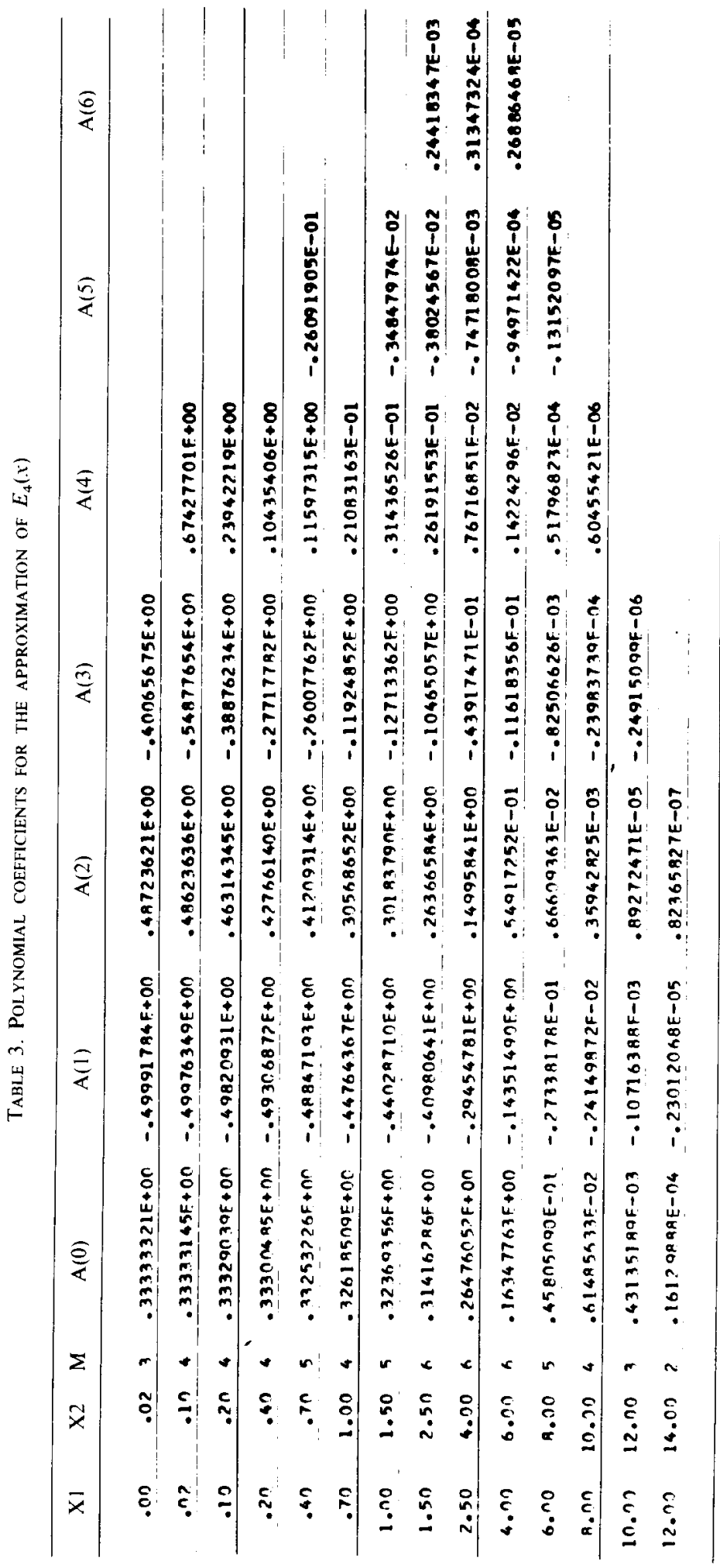


The coefficients were used in a subroutine run on an IBM 7090 computer. With the exception of the region near $x=0.0$ for $E_{2}(x)$, the average time for evaluation was about $0.24 \mathrm{~m} \mathrm{sec}$, almost half the average time of the system subroutine for the exponential function. A Fortran IV listing of the subroutines may be obtained from the author.

Acknowledgement This work was supported by NSF under Cirant No. GP-4.385 to the Department of Meteorology and Oceanography. University of Michigan.

\section{REFERENCES}

1. M. Albramowitz and 1. A. Strgilin. Handhook of Mathematical Finctions. 1! S. Government Printing Office. Washington (1964).

2. F. E. Harris. Math. Tables Aids Comp. 11, 9 (1957).

3. J. Muli.F and P. Hurst, Math. Tahles Aids Comp. 12, 187 (1958). 